\title{
EXISTENCE AND UNIQUENESS FOR A CRYSTALLINE MEAN CURVATURE FLOW
}

\author{
ANTONIN CHAMBOLLE, MASSIMILIANO MORINI, AND MARCELLO PONSIGLIONE
}

\begin{abstract}
An existence and uniqueness result, up to fattening, for a class of crystalline mean curvature flows with natural mobility is proved. The results are valid in any dimension and for arbitrary, possibly unbounded, initial closed sets. The comparison principle is obtained by means of a suitable weak formulation of the flow, while the existence of a global-in-time solution follows via a minimizing movements approach.

Keywords: Geometric evolution equations, Minimizing movements, Crystalline mean curvature motion.
\end{abstract}

2000 Mathematics Subject Classification: 53C44, 49M25, 35D40.

\section{INTRODUCTION}

In this paper we deal with the anisotropic mean curvature motion; that is, flows of sets $t \mapsto E(t)$ (formally) governed by the law

$$
V(x, t)=-m\left(\nu^{E(t)}\right) \kappa_{\phi}^{E(t)}(x),
$$

where $V(x, t)$ stands for the (outer) normal velocity of the boundary $\partial E(t)$ at $x, \phi$ is a given norm on $\mathbb{R}^{N}$ representing the surface tension, $\kappa_{\phi}^{E(t)}$ is the anisotropic mean curvature of $\partial E(t)$ associated with the anisotropy $\phi$, and $m$ is a positive mobility which depends on the outer unit normal $\nu^{E(t)}$ to $\partial E(t)$. Such an evolution law may be regarded as the gradient flow (with respect to a suitable formal Riemannian structure) of the anisotropic perimeter functional

$$
P_{\phi}(E)=\int_{\partial E} \phi\left(\nu^{E}\right) d \mathcal{H}^{N-1},
$$

the anisotropic curvature $\kappa_{\phi}^{E}$ of $\partial E$ being nothing but the first variation of (1.2) at $E$. When $\phi$ is differentiable in $\mathbb{R}^{N} \backslash\{0\}$, then $\kappa_{\phi}^{E}$ is given by

$$
\kappa_{\phi}^{E}=\operatorname{div}\left(\nabla \phi\left(\nu^{E}\right)\right) \text {. }
$$

However, we are particularly interested in the case when $\phi$ is not differentiable, for instance the crystalline case, when the unit ball $B_{\phi}:=\{\phi \leq 1\}$, known as the Frank diagram, is a convex polytope. In the latter case, we will only consider the natural mobility given by $m=\phi$. With this choice, (1.1) has the interesting property that the flow starting from a Wulff shape, that is, a level set of the polar $\phi^{\circ}$ of $\phi$, consists in a one-parameter family of shrinking Wulff shapes that extinguish in finite time. We recall that Wulff shapes are the only solutions to the isoperimetric problem associated with $P_{\phi}$ (see [26]). 
The law (1.1) is used to describe several phenomena in Materials Science and Crystal Growth, see for instance [40, 33. From the mathematical point of view, the geometric motion is well defined in a classical sense in the smooth case, that is, when $\phi$ is at least $C^{3, \alpha}$ (as well as the initial surface, and except at the origin) and "elliptic" (which means for instance that $\phi^{2}$ is strongly convex) [2]. Of course, the classical mean curvature flow falls within this class and corresponds to the choice $\phi=$ Euclidean norm. In the smooth case, the main mathematical difficulties are related to the fact that singularities (like pinching) may form in finite time (see for instance [32]) in dimensions $N \geq 3$. Thus, the strong formulation of (1.1), which requires smoothness of the evolving sets, is well defined only for short times and one needs a weaker notion of solution that can handle the presence of singularities in order to define the flow for all positive times. When $\phi$ is smooth, this task has been already accomplished and different approaches have been proposed in the literature, starting from the pioneering work by Brakke [15, who suggested a weak formulation of the motion by mean curvature yielding deep regularity results but lacking uniqueness. These uniqueness issues have been subsequently overcome via the so-called level set approach [38, 24, 21. In particular, the case of (1.1) for $m, \phi$ of class $C^{2}$ is covered by [21]. The main idea is to represent the initial set as the zero sublevel set of a function $u_{0}$ and then to let all these level sets evolve according to the same geometric law (which makes sense thanks to the fact that the evolutions which we consider preserve inclusion). This procedure defines a time-dependent function $u(x, t)$ and transforms the geometric equation into a (degenerate) parabolic equation for $u$, which is shown to admit a unique viscosity solution with the prescribed initial datum $u_{0}$. The evolution of the zero sublevel set of such a solution defines a generalized motion (see also [11]), which exists for all times and agrees with the classical one for short times, before the appearance of singularities (see [25]). Such a motion satisfies a comparison principle and is unique whenever the level sets of $u$ have zero Lebesgue measure, i.e., whenever the so-called fattening phenomenon does not occurs. Fattening may in fact appear even for a smooth initial datum $E^{0}$ (see [7]), but its occurrence is in some sense very "rare": for instance, it is easy to understand that almost all the sublevels sets of the signed distance function from any given set $E^{0}$ will not generate any fattening.

A third approach is represented by the minimizing movements scheme devised by Almgren, Taylor and Wang [2] and Luckhaus and Sturzenhecker [36]. It consists in constructing a sequence of discrete-in-time evolutions by iteratively solving suitable incremental minimum problems. Any limit of these evolutions as the time step vanishes defines a motion, which exists for all positive times (and is shown to be Hölder-continuous in time for the $L^{1}$ norm). The connections between the generalized level set motion and Brakke solutions has been investigated in 34. A simple proof of convergence of the Almgrem-Taylor-Wang (ATW) to the generalized motion is shown in [20], while a consistency result was already shown in [2]. See also 22] for a similar convergence proof in a more general setting (allowing for unbounded surfaces, as in the present paper), and 35] for new proofs and a generalization to partitions. Roughly speaking, it turns out that whenever fattening 
does not occur, the generalized level set motion coincides with the ATW flow and is also a solution in the sense of Brakke.

Let us now consider a crystalline anisotropy. This case is more difficult, due to the lack of smoothness in the involved differential operators. Indeed, the crystalline normal $\nabla \phi\left(\nu^{E}\right)$ is not uniquely defined for some directions and one needs to look at suitable selections of the (multivalued) subdifferential map, that is, vector fields $z: \partial E \rightarrow \mathbb{R}^{N}$, such that $z(x) \in \partial \phi\left(\nu^{E}(x)\right)$ for a.e. $x$. If there exists an admissible field $z$ with tangential divergence $\operatorname{div}_{\tau} z$ in $L^{2}(\partial E)$, then the crystalline curvature is given by the tangential divergence of $z$, where $\operatorname{div}_{\tau} z$ has minimal $L^{2}$-norm among all admissible fields (see [13, 29]). In particular, the crystalline curvature has a nonlocal character.

Showing (even local-in-time) existence and uniqueness for crystalline mean curvature flows is somewhat harder and still largely open. Only in dimension 2, the problem has been settled by developing a crystalline version of the viscosity approach for the level-set equation, see [28. If the initial set is itself an appropriate planar crystal, the evolution equation boils down to a system of ODEs which has been studied in many former works, see in particular [3, 8, 9, 27, 30, while existence and uniqueness of strong solutions for initial "regular" (in an appropriate sense) sets was shown recently in [17. One advantage of the level-set approach of [28 is the ability to address much more general equations where the speed depends on the crystalline curvature and the normal in an non-linear way.

In dimensions $N \geq 3$, the only general available notion of global-in-time solution we are aware of is the minimizing movements motion provided by the ATW scheme; however, no general comparison results have been established so far. In fact, the higher-dimensional uniqueness results we know of deal with special classes of initial data (for instance convex initial data as in [16, 14, or polyhedral sets as in [31]) or with very specific anisotropies (see [29] where a comparison principle valid in all dimensions has been established for the anisotropy $\phi(\nu)=\left|\nu^{\prime}\right|+\left|\nu_{N}\right|$, with $\nu_{N}:=\nu \cdot e_{N}$ and $\left|\nu^{\prime}\right|$ the Euclidean norm of the orthogonal projection of $\nu$ onto $\left.e_{N}^{\perp}\right)$. However, Y. Giga has recently announced a very general existence and uniqueness result in the viscosity sense in dimension $N=3$.

In this paper we prove a global-in-time existence and uniqueness (up to possible fattening) result for the crystalline mean curvature flow valid in all dimensions, for arbitrary (possibly unbounded) initial sets, and for general crystalline anisotropies $\phi$, but under the particular choice $m=\phi$ in (1.1). We do so by providing a suitable weak formulation of the problem and then by showing that such a notion yields a comparison principle. We then implement a variant of the ATW scheme to establish an existence result.

Le us describe our approach in more details. It is based on ideas of [39, 6]. In order to motivate our formulation, let us assume for a moment that $\phi$ is smooth and that $t \mapsto E(t)$ is a regular flow obeying (1.1). Set $d(\cdot, t):=\operatorname{dist}(\cdot, E(t))$, where dist denotes the distance induced by the polar norm $\phi^{\circ}$ (see (2.1) and (2.3) below). Then it is easy to see that the time partial derivative $\partial_{t} d$ of $d$ on $\partial E(t)$ equals $-V / \phi\left(\nu^{E(t)}\right)$, with $V$ denoting the outer normal velocity of the moving boundary. On the other hand, this quantity $V / \phi\left(\nu^{E(t)}\right)$ is nothing else as the speed of the 
moving boundary along the Cahn-Hoffmann normal $\nabla \phi\left(\nu^{E(t)}\right)$, see 33, 13. Thus, (1.1) may be rewritten as

$$
\partial_{t} d=\kappa_{\phi}^{E(t)}=\operatorname{div}(\nabla \phi(\nabla d)) \quad \text { on } \partial E(t)=\partial\{d(\cdot, t)=0\} .
$$

(Here and throughout the paper $\nabla$ stands for the spatial gradient.) On the other hand, if we look at a positive $s$-level set of $d$, the (weighted) normal velocity of $x \in\{\operatorname{dist}(\cdot, t)=s\}$ equals the normal velocity of its projection $y$ on $\partial E(t)$, which is given by the anisotropic curvature $\kappa_{\phi}^{E(t)}(y)$ of $\partial E(t)$ at $y$. Since (as long as the surfaces are smooth)

$$
\kappa_{\phi}^{\{d(\cdot, t)=s\}}(x)=\operatorname{div}(\nabla \phi(\nabla d))(x, t) \leq \kappa_{\phi}^{E(t)}(y),
$$

we deduce that

$$
\partial_{t} d \geq \operatorname{div}(\nabla \phi(\nabla d)) \quad \text { in }\{d>0\}
$$

as long as $E(\cdot)$ is nonempty. In words, the positive level sets of the distance function shrink with a velocity which is higher than that given by the anisotropic curvature, and thus they may be regarded as super-flows or supersolutions of the geometric motion. Analogously, setting $d^{c}(\cdot, t):=\operatorname{dist}\left(\cdot, E^{c}(t)\right)$, where $E^{c}$ stands for the complement of $E$, we have

$$
\partial_{t} d^{c} \geq \operatorname{div}\left(\nabla \phi\left(\nabla d^{c}\right)\right) \quad \text { in }\left\{d^{c}>0\right\}
$$

as long as $E^{c}(\cdot)$ is nonempty. We may conclude that a smooth flow $t \mapsto E(t)$ of sets solves (1.1) if and only if (1.4) and (1.5) are satisfied.

As already remarked before, when $\phi$ is crystalline $\nabla \phi(\nabla d)$ may not be defined and must be replaced in general by a suitable selection of the subdifferential map, that is, by a vector-field $z \in L^{\infty}\left(\{d>0\} ; \mathbb{R}^{N}\right)$ such that $z(x) \in \partial \phi(\nabla d(x))$ for a.e. $x$, where $\partial \phi$ denotes the subdifferential of $\phi$. Any such $z$ will be called admissible for $d$.

The above discussion motivates the following weak formulation of the crystalline flow: we will say that a one-parameter family $t \mapsto E(t)$ of closed sets, satisfying suitable continuity properties (see Definition 2.1 below) is a weak supersolution of (1.1) with initial datum $E^{0}$ if $E(0) \subseteq E^{0}$ and there exists a vector-field $z$, admissible for $d$, such that (1.4) hold in the sense of distributions, with $\nabla \phi(\nabla d)$ replaced by $z$. We will say instead that $t \mapsto E(t)$ is a weak-subsolution of (1.1) if $E(0) \supseteq E^{0}$ and $t \mapsto(\stackrel{\circ}{E}(t))^{c}$ is weak supersolution. Finally, we will say that $t \mapsto E(t)$ is a weak solution if it is both a weak sub- and a supersolution (with initial datum $E^{0}$ ). Mostly for technical reasons, we will require in addition that the positive part of $\operatorname{div} z$ is bounded in $\{d \geq \delta\}$ for all $\delta>0$.

Let us notice that this formulation of the curvature flow in terms of the distance function has been already exploited for the standard mean curvature motion and its regular anisotropic variants. In fact, it is close in spirit to the distance formulation proposed and studied in [39, although it is somewhat stronger as it require the differential inequalities to hold in a distributional sense, rather than in the viscosity sense considered in [39]. In this respect, our formulation is reminiscent of the approach developed in [16]. 
We now describe the plan of the paper. In Section 2 after recalling some preliminaries definitions and introducing the main notation, we give the precise weak formulation of the sub- and supersolutions to the anisotropic mean curvature flow. In Section 3 we establish a comparison principle between sub- and supersolutions, which by standard arguments yields the uniqueness of the crystalline flow whenever fattening does not occur. We remark that the distributional formulation described above allows for a proof of the comparison, which is closer in spirit to the uniqueness proofs for standard parabolic equations. In particular, our argument is more elementary than the typical "viscosity" proof that is based on delicate regularization procedures and fine differentiability properties of semiconvex functions. In Section 4 we provide an existence results for the the weak formulation of the crystalline flow, which is based on the reformulation of the minimizing movements scheme of Almgren-Taylor-Wang / Luckhaus-Sturzenhecker introduced in [18, 16]. Such a variant can be considered as a combination of the ideas of [2] and the threshold dynamics algorithm studied in [23], and has several advantages: for instance, it makes it easier to establish a comparison principle for the discrete-in-time evolutions and it works equally well for bounded and unbounded sets (as already exploited in [22]). In the main theorem of the section, we establish the convergence of the minimizing movements scheme to a weak solution, whenever no fattening occurs.

We conclude this introduction by commenting on the restriction $m=\phi$ in (1.1). Although such a mobility is rather natural (for instance it forces Wulff shapes to evolve in a self-similar way), it is not the most general case and different mobilities could be considered as physically interesting. However, at the moment, in the crystalline case we are able to provide the right convergence estimates for the minimizing movements scheme only under this assumption; the main technical reason is related to the fact that if dist is the distance induced by the polar norm $\phi^{\circ}$, then the crystalline curvatures of the positive level sets of $\operatorname{dist}(\cdot, E)$ are bounded above (this can be easily understood since in this case the sublevel sets of $\operatorname{dist}(\cdot, E)$ admit an inner tangent Wulff shape at all points of the boundary). Nevertheless, we remark that in the case of a smooth elliptic anisotropy, all our results and methods would work with any mobility $m$, thus showing that the viscosity solutions already studied in [24, 21, 39] satisfy in fact a stronger (distributional) formulation. The extension of our results to more general mobilities in the crystalline case will be the subject of future investigations.

\section{ACKNOWLEDGEMENTS}

Part of this research was done in the Institut Henri Poincaré in Paris, where M. Morini and M. Ponsiglione were hosted for a month in 2015 thanks to the "Research in Paris" programme of this mathematical institute. The authors are very grateful for this support. In addition, A. Chambolle was partially supported by the ANR, programs ANR-12-BS01-0014-01 "GEOMETRYA" and ANR-12-BS01-000801 "HJnet". 


\section{A WEAK FORMUlation of THE CRYSTALLine MEAN CURVATURE FLOW}

In this section we introduce a suitable weak formulation of the crystalline mean curvature flow. Such a notion of solution resembles the formulation due to [39]. However, here we will not consider the viscosity setting of [39] and we will rather be concerned with distributional solutions (which appear for instance in [16]).

2.1. Preliminaries. In this subsection we introduce the main objects and notation used throughout the paper.

Let $\phi$ denote a fixed norm on $\mathbb{R}^{N}$, that is, a convex, even and 1-homogeneous real-valued function, which will play the role of the anisotropic interfacial energy density. In the terminology of crystal growth this is also called surface tension. Note that we do not assume any further regularity on $\phi$ and in fact the main case of interest is when $\phi$ is crystalline, that is, when the associated unit ball is a convex polytope. The interfacial energy is then given by

$$
P_{\phi}(E):=\sup \left\{\int_{E} \operatorname{div} \zeta d x: \zeta \in C_{c}^{1}\left(\mathbb{R}^{N} ; \mathbb{R}^{N}\right), \phi^{\circ}(\zeta) \leq 1\right\},
$$

where we recall that the polar norm $\phi^{\circ}$ is defined as

$$
\phi^{\circ}(\xi):=\sup _{\phi(\eta) \leq 1} \eta \cdot \xi
$$

It can be checked that $P_{\phi}(E)$ is finite if and only if $E$ is a set of finite perimeter and, in this case,

$$
P_{\phi}(E)=\int_{\partial^{*} E} \phi\left(\nu^{E}\right) d \mathcal{H}^{N-1},
$$

where $\partial^{*} E$ denotes the so-called reduced boundary of $E$ (see for instance [5]). More generally, given a function $u \in B V_{l o c}\left(\mathbb{R}^{N}\right)$ we may consider the anisotropic total variation maesure of $u$, which on the open (bounded if $u \notin B V\left(\mathbb{R}^{N}\right)$ ) subsets $\Omega \subset \mathbb{R}^{N}$ is defined as

$$
\phi(D u)(\Omega):=\sup \left\{\int_{\Omega} u \operatorname{div} \zeta d x: \zeta \in C_{c}^{1}\left(\Omega ; \mathbb{R}^{N}\right), \phi^{\circ}(\zeta) \leq 1\right\} .
$$

Because of the homogeneity of $\phi$ it turns out that $\phi(D u)$ coincides with the nonnegative Radon measure in $\mathbb{R}^{N}$ given by $\nabla u d x+\phi\left(\frac{D^{s} u}{\left|D^{s} u\right|}\right)\left|D^{s} u\right|$, where $\nabla u$ stands for the absolutely continuous part of $D u$ and $\frac{D^{s} u}{\left|D^{s} u\right|}$ denotes the Radon-Nykodim derivative of the singular part $D^{s} u$ of $D u$ with respect to its (isotropic) total variation $\left|D^{s} u\right|$, see $[5]$.

Among the important properties of $\phi$ and $\phi^{\circ}$ let us mention the fact that $\partial \phi(0)=$ $\left\{\xi: \phi^{\circ}(\xi) \leq 1\right\}$ while $\partial \phi^{\circ}(0)=\{\xi: \phi(\xi) \leq 1\}$. Moreover, for $\eta \neq 0$

$$
\partial \phi(\eta)=\left\{\xi: \phi^{\circ}(\xi) \leq 1 \text { and } \xi \cdot \eta=\phi(\eta)\right\}=\left\{\xi: \phi^{\circ}(\xi)=1 \text { and } \xi \cdot \eta=\phi(\eta)\right\}
$$

(and the symmetric statement for $\phi^{\circ}$ ). An easy consequence of the above characterization is that if $\eta \in \partial \phi^{\circ}(x)$ and $x \neq 0$, then $x / \phi^{\circ}(x) \in \partial \phi(\eta)$.

The set

$$
W(0,1):=\left\{y: \phi^{\circ}(y) \leq 1\right\}
$$

is called the Wulff shape associated with $\phi$. More generally, for $x \in \mathbb{R}^{N}$ and $R>0$, we will denote by

$$
W(x, R):=\left\{y: \phi^{\circ}(y-x) \leq R\right\}
$$


the Wulff shape of radius $R$ and center $x$. In the Finsler metric framework associated with $\phi^{\circ}$, Wulff shapes play the same role as standard balls do in the Euclidean setting. In particular, it is well-known that $W(0, R)$ is the unique (up to translations) solution of the anisotropic isoperimetric problem

$$
\min \left\{P_{\phi}(E):|E|=|W(0, R)|\right\},
$$

see for instance [26].

Given a set $E \subseteq \mathbb{R}^{N}$, we denote by $\operatorname{dist}(\cdot, E)$ the distance from $E$ induced by $\phi^{\circ}$, that is, for any $x \in \mathbb{R}^{N}$

$$
\operatorname{dist}(x, E):=\inf _{y \in E} \phi^{\circ}(x-y)
$$

if $E \neq \emptyset$ and $\operatorname{dist}(x, \emptyset):=+\infty$. Moreover, we denote by $d_{E}$ the signed distance from $E$ induced by $\phi^{\circ}$, i.e.,

$$
d_{E}(x):=\operatorname{dist}(x, E)-\operatorname{dist}\left(x, E^{c}\right)
$$

so that $\operatorname{dist}(x, E)=d_{E}(x)^{+}$and $\operatorname{dist}\left(x, E^{c}\right)=d_{E}(x)^{-}$(here and throughout the paper we adopt the standard notation $t^{+}:=t \vee 0$ and $\left.t^{-}:=(-t)^{+}\right)$. Note that $\phi\left(\nabla d_{E}\right)=1$ a.e. in $\mathbb{R}^{N} \backslash \partial E$.

We finally recall the notion of Kuratowski convergence. We say that a sequence of closed sets $E_{n}$ in $\mathbb{R}^{m}$ converges to a closed set $E$ in the Kuratowki sense, and we write

$$
E_{n} \stackrel{\mathcal{K}}{\longrightarrow} E
$$

if the following conditions are satisfied:

(i) if $x_{n} \in E_{n}$, any limit point of $\left\{x_{n}\right\}$ belongs to $E$;

(ii) any $x \in E$ is the limit of a sequence $\left\{x_{n}\right\}$, with $x_{n} \in E_{n}$.

One can easily see that $E_{n} \stackrel{\mathcal{K}}{\longrightarrow} E$ if and only if $\operatorname{dist}\left(\cdot, E_{n}\right) \rightarrow \operatorname{dist}(\cdot, E)$ locally uniformly in $\mathbb{R}^{m}$ (here one may consider the distance associated to any norm). In particular, by the Ascoli-Arzelà Theorem, any sequence of closed sets admits a subsequence which converges in the Kuratowski sense.

2.2. A weak formulation of the crystalline flow. In this subsection we introduce the weak formulation of the crystalline flow we will deal with. We refer the reader to the introduction for the motivation behind this definition.

Definition 2.1. Let $E^{0} \subset \mathbb{R}^{N}$ be a closed set. Let $E$ be a closed set in $\mathbb{R}^{N} \times[0,+\infty)$ and for each $t \geq 0$ denote $E(t):=\left\{x \in \mathbb{R}^{N}:(x, t) \in E\right\}$. We say that $E$ is a supersolution of the curvature flow (1.1) with initial datum $E^{0}$ if

(a) $E(0) \subseteq E^{0}$;

(b) for all $t \geq 0$ if $E(t)=\emptyset$, then $E(s)=\emptyset$ for all $s>t$;

(c) $E(s) \stackrel{\mathcal{K}}{\longrightarrow} E(t)$ as $s \nearrow t$ for all $t>0$ (left-continuity);

(d) $\operatorname{setting} d(x, t):=\operatorname{dist}(x, E(t))$ for $(x, t) \in \mathbb{R}^{N} \times\left(0, T^{*}\right) \backslash E$ and

$$
T^{*}:=\inf \{t>0: E(s)=\emptyset \text { for } s \geq t\},
$$

then the inequality

$$
\partial_{t} d \geq \operatorname{div} z
$$


holds in the distributional sense in $\mathbb{R}^{N} \times\left(0, T^{*}\right) \backslash E$ for a suitable $z \in$ $L^{\infty}\left(\mathbb{R}^{N} \times\left(0, T^{*}\right)\right)$ such that $z \in \partial \phi(\nabla d)$ a.e., $\operatorname{div} z$ is a Radon measure in $\mathbb{R}^{N} \times\left(0, T^{*}\right) \backslash E$, and $(\operatorname{div} z)^{+} \in L^{\infty}\left(\left\{(x, t) \in \mathbb{R}^{N} \times\left(0, T^{*}\right): d(x, t) \geq \delta\right\}\right)$ for every $\delta>0$.

We say that $A$, open set in $\mathbb{R}^{N} \times[0,+\infty)$, is a subsolution with initial datum $E^{0}$ if $A^{c}$ is a supersolution with initial datum $\left(\stackrel{\circ}{E}^{0}\right)^{c}$.

Finally, we say that $E$, closed set in $\mathbb{R}^{N} \times[0,+\infty)$, is a solution with initial datum $E^{0}$ if it is a supersolution and if $\stackrel{\circ}{E}$ is a subsolution, both with initial datum $E^{0}$.

Remark 2.2. Notice that the initial condition for supersolutions may be rewritten as $\stackrel{\circ}{E}^{0} \subseteq A(0)$. In particular, if $\partial E^{0}=\partial \stackrel{\circ}{E}^{0}$ and $E$ is a solution according to the previous definition, then $E(0)=E^{0}$.

Remark 2.3. If $\phi$ is $C^{2}$, then one can check that this definition is stronger than the definition in the viscosity sense (see in particular [39, 11]).

We start by observing some useful continuity properties of the map $d$ introduced in the previous definition.

Lemma 2.4. Let $E$ be a supersolution. Then, for each $t \in\left[0, T^{*}\right), d(\cdot, s)$ converges locally uniformly in $\{x: d(x, t)>0\}$ as $s \searrow t$ to for some function $d^{r}$ with $d^{r} \geq$ $d(\cdot, t)$ in $\{x: d(x, t)>0\}$.

Remark 2.5. Observe that by condition (c) in the definition (which is mostly technical and forbids artificial constructions such as a supersolution which jumps to $E(t)=\mathbb{R}^{N}$ at a given time $\left.t>0\right), t \mapsto d(\cdot, t):=d(\cdot, E(t))$ is left-continuous with respect to the local uniform convergence.

Proof. By condition (d) of Definition 2.1, the distributional derivative $\partial_{t} d$ is a Radon measure in $\mathbb{R}^{N} \times\left(0, T^{*}\right) \backslash E$, so that $d$ is locally a function with bounded variation in this (open) domain. In particular, for a.e. $x \in \mathbb{R}^{N}$ the map $s \mapsto d(x, s)$ has a right limit $d^{r}(x, t)$ at each time $t \in\left[0, T^{*}\right)$ such that $d(x, t)>0$. Since the functions $d(\cdot, s)$ are also equi-Lipschitz in space as $s$ varies, we may conclude that the right limit is in fact locally uniform in $\{x: d(x, t)>0\}$.

Since $E$ is closed, for every $t \in\left[0, T^{*}\right]$ we clearly have that all Kuratowski cluster points of $E(s)$ as $s \rightarrow t$ are contained in $E(t)$, equivalently, $d(x, t) \leq$ $\liminf _{s \rightarrow t} d(x, s)$. Thus, $d^{r} \geq d(\cdot, t)$ in $\{x: d(x, t)>0\}$.

\section{Comparison RESUlts}

In this section we prove the main comparison principle between sub- and supersolutions (see Theorem 3.3). In Lemma 3.2 below, we establish a first (suboptimal) comparison result between a supersolution and a suitable anisotropic total variation flow (see [12, 37]). To this aim, we give an explicit solution to the anisotropic total variation flow with initial datum $\phi^{\circ}$.

Lemma 3.1. The pair $(f, \zeta)$ defined by

$$
f(x, t):= \begin{cases}r(t)+t \frac{N-1}{r(t)} & \text { if } \phi^{\circ}(x) \leq r(t):=\sqrt{(N+1) t} \\ \phi^{\circ}(x)+t \frac{N-1}{\phi^{\circ}(x)} & \text { otherwise }\end{cases}
$$


and

$$
\zeta(x, t):= \begin{cases}\frac{x}{r(t)} & \text { if } \phi^{\circ}(x) \leq r(t), \\ \frac{x}{\phi^{\circ}(x)} & \text { if } \phi^{\circ}(x) \geq r(t),\end{cases}
$$

solve the following Cauchy problem for the $\phi$-total variation flow in $\mathbb{R}^{N}$ :

$$
\begin{cases}\partial_{t} f=\operatorname{div} \zeta & \text { a.e. in } \mathbb{R}^{N} \times(0,+\infty), \\ \zeta \in \partial \phi(\nabla f) & \text { a.e. in } \mathbb{R}^{N} \times(0,+\infty), \\ f(\cdot, 0)=\phi^{\circ} . & \end{cases}
$$

Moreover, given $\lambda>1$, the pair $\left(f_{\lambda}, \zeta_{\lambda}\right)$ given by

$$
f_{\lambda}(x, t):=\lambda f(x, t / \lambda) \quad \zeta_{\lambda}(x, t):=\zeta(x, t / \lambda)
$$

for $(x, t) \in \mathbb{R}^{N} \times(0,+\infty)$ solves (3.3), with the initial datum $\phi^{\circ}$ replaced by $\lambda \phi^{\circ}$.

Proof. Recalling that $\zeta \in \partial \phi(\nabla f)$ is equivalent to $\phi^{\circ}(\zeta) \leq 1, \zeta \cdot \nabla f=\phi(\nabla f)$ (see $(2.2)$ ), the proof follows by direct verification. The details are left to the reader.

Next lemma provides a first comparison estimate, which is far from being sharp. However, the optimal estimate can be established a posteriori as a consequence of our main comparison theorem (see Theorem 3.3 below).

Lemma 3.2. Let $E$ be a supersolution and $d:=\operatorname{dist}(\cdot, E(\cdot))$ the associated one parameter family of distance functions. Assume that for some $(\bar{x}, \bar{t}) \in \mathbb{R}^{N} \times[0,+\infty)$ we have $d(\bar{x}, \bar{t}) \geq R>0$. Then, there exists a constant $\chi_{N}>0$ such that $d(\bar{x}, \bar{t}+s) \geq$ $R-\chi_{N} \sqrt{s}$ for all $s \in\left[0, R^{2} /\left(16 \chi_{N}^{2}\right)\right]$.

Proof. Observe first that thanks to Lemma 2.4 since $d(\cdot, \bar{t}) \geq R / 4$ in $\left\{x: \phi^{\circ}(x-\right.$ $\bar{x}) \leq 3 R / 4\}=W(\bar{x}, 3 R / 4)$, there exists a (unknown) time $t^{*}$ such that $d(\cdot, \bar{t}+s)>$ $\alpha>0$ in $W(\bar{x}, 3 R / 4)$ for all $s \in\left[0, t^{*}\right]$ for some positive $\alpha$. We will compare $d$ with the solution $\delta$ of the $\phi$-total variation flow starting from

$$
\delta(\cdot, 0):=R-\frac{4}{3} \phi^{\circ}(\cdot-\bar{x}) .
$$

More precisely, setting $\delta(x, s):=R-f_{4 / 3}(x-\bar{x}, s)$, where $f_{4 / 3}(x, t):=4 / 3 f(x, 3 t / 4)$ and $f$ is given by (3.1), by Lemma 3.1$]$ satisfies

$$
\begin{cases}\partial_{t} \delta=\operatorname{div} \xi & \text { in } \mathbb{R}^{N} \times(0,+\infty), \\ \xi \in \partial \phi(\nabla \delta) & \text { a.e. in } \mathbb{R}^{N} \times(0,+\infty),\end{cases}
$$

where $\xi(x, t)=-\zeta(x, 3 t / 4)$, with $\zeta$ defined by (3.2). Note that $\delta$ is negative outside $W(\bar{x}, 3 R / 4)$ for all positive times.

Let $\Psi(s)$ be a smooth, convex, nonnegative function, which vanishes only for $s \leq 0$, and consider the function $w(x, s):=\Psi(\delta(x, s)-d(x, \bar{t}+s))$. Without loss of generality, we assume to simplify the notation that $\bar{t}=0$. By construction, $w(x, 0) \equiv 0$ in $W(\bar{x}, 3 R / 4)$ and $w(\cdot, s) \equiv 0$ on $\partial W(\bar{x}, 3 R / 4)$ for $0 \leq s \leq t^{*}$.

Since $\phi(\nabla d) \leq 1$ a.e. and $\partial_{t} d$ is a measure wherever it is positive, it follows that $d$ is a function in $B V_{l o c}\left(W(\bar{x}, 3 R / 4) \times\left(0, t^{*}\right)\right)$ and its distributional time derivative has the form

$$
\partial_{t} d=\sum_{t \in J}[d(\cdot, t+0)-d(\cdot, t-0)] d x+\partial_{t}^{d} d
$$


where $J$ is the (countable) set of times where $d$ jumps and $\partial_{t}^{d} d$ is the diffuse (Cantor+absolutely continuous) part of the derivative. It turns out that (Lemma 2.4) $d(\cdot, t+0)-d(\cdot, t-0) \geq 0$ for each $t \in J$. Moreover, since the positive part of $\operatorname{div} z$ is absolutely continuous with respect to the Lebesque measure, (2.4) entails

$$
\partial_{t}^{d} d \geq \operatorname{div} z .
$$

Using the chain rule for $B V$ functions, see [4]), one has

$$
\begin{aligned}
\partial_{t} w=\sum_{t \in J}[\Psi(\delta(\cdot, t)-d(\cdot, t+0))-\Psi(\delta(\cdot, t)-d(\cdot, t-0))] d x & \\
& +\Psi^{\prime}(\delta-d)\left(\partial_{t} \delta-\partial_{t}^{d} d\right) \leq \Psi^{\prime}(\delta-d)(\operatorname{div} \xi-\operatorname{div} z) .
\end{aligned}
$$

Hence, for a.e. $t \leq t^{*}$, using the fact that $\phi$ and $\Psi$ are convex, $\Psi^{\prime}(\delta-d)$ vanishes on $\partial W(\bar{x}, 3 R / 4)$ and recalling (3.4), we have

$$
\begin{aligned}
\partial_{t} \int_{W(\bar{x}, 3 R / 4)} w d x \leq \int_{W(\bar{x}, 3 R / 4)} & \Psi^{\prime}(\delta-d)(\operatorname{div} \xi-\operatorname{div} z) \\
= & -\int_{W(\bar{x}, 3 R / 4)}(\xi-z) \cdot(\nabla \delta-\nabla d) \Psi^{\prime \prime}(\delta-d) \leq 0 .
\end{aligned}
$$

It follows that $w=\Psi(\delta-d)=0$, that is, $d \geq \delta$ a.e. at all times less than $t^{*}$. More precisely, for $0 \leq s \leq t^{*}$ we have

$$
d(\bar{x}, \bar{t}+s) \geq R-f_{4 / 3}(x-\bar{x}, s)=R-\frac{4 N}{\sqrt{3}} \sqrt{\frac{s}{N+1}}=: R-\chi_{N} \sqrt{s} .
$$

It follows from (3.5) that $d(\bar{x}, \bar{t}+s)>3 R / 4$ and, in turn, $d(\cdot, \bar{t}+s)>0$ on $\partial W(\bar{x}, 3 R / 4)$ for all $s<\min \left\{t^{*}, R^{2} /\left(16 \chi_{N}^{2}\right)\right\}$. But then we can restart the argument above to find that (3.5) remains valid for slightly larger times. Thus, we may conclude that (3.5) holds at least for all $0 \leq s \leq R^{2} /\left(16 \chi_{N}^{2}\right)$. This concludes the proof of the lemma.

Now we can state the main result of this section, which is a comparison result between sub- and supersolutions.

Theorem 3.3. Let $E$ be a supersolution with initial datum $E^{0}$ and $F$ be a subsolution with initial datum $F^{0}$. Assume that $\operatorname{dist}\left(E^{0}, F^{0^{c}}\right)=: \Delta>0$. Then for each $t \geq 0, \operatorname{dist}\left(E(t), F^{c}(t)\right) \geq \Delta$.

Proof. Let $T_{E}^{*}$ and $T_{F}^{*}$ be the maximal existence time for $E$ and $F$. For all $t>$ $\min \left\{T_{E}^{*}, T_{F}^{*}\right\}$ we have that either $E$ or $F^{c}$ is empty. In this case, clearly the conclusion holds true.

Now, consider the case $t \leq \min \left\{T_{E}^{*}, T_{F}^{*}\right\}$ (and assume without lost of generality that $\left.T_{E}^{*}, T_{F}^{*}>0\right)$. Let us fix $0<\eta_{1}<\eta_{1}^{\prime}<\eta_{1}^{\prime \prime}<\eta_{2}^{\prime \prime}<\eta_{2}^{\prime}<\eta_{2}<\Delta$. We will show the conclusion of the theorem for a time interval $\left(0, t^{*}\right)$ for a suitable $t^{*}$ depending only on $\eta_{1}, \eta_{1}^{\prime}, \eta_{1}^{\prime \prime}, \eta_{2}^{\prime \prime}, \eta_{2}^{\prime}, \eta_{2}$, and ultimately only on $\Delta$. It is clear then that reiterating the argument yields the conclusion of the theorem for all times. We recall that $d_{E}(x, t):=\operatorname{dist}(x, E(t))-\operatorname{dist}\left(x, E^{c}(t)\right)$ and $d_{F}$ is defined analogously. We denote by $z_{E}$ and $z_{F}$ the fields appearing in the definition of 
super- and subsolutions (see Definition 2.1), corresponding to $E$ and $F$, respectively. Define

$$
S:=\left\{x \in \mathbb{R}^{N}: \eta_{1}<d_{E}(x, 0)<\eta_{2}\right\}
$$

and note that by Lemma 3.2 there exists $t^{*}>0$ depending only on $\eta_{1}, \Delta-\eta_{2}$ such that

$$
d_{E}(x, t) \geq d_{E}(x, 0)-\chi_{N} \sqrt{t} \quad \text { for all } x \in \bar{S} \text { and } t \in\left(0, t^{*}\right) \text {. }
$$

We now set

$$
\begin{aligned}
& \tilde{d}_{E}:=d_{E} \vee\left(\eta_{1}^{\prime}+\chi_{N} \sqrt{t}\right), \\
& \tilde{d}_{F}:=\left(d_{F}+\Delta\right) \wedge\left(\eta_{2}^{\prime}-\chi_{N} \sqrt{t}\right) .
\end{aligned}
$$

Clearly, by our assumptions $\tilde{d}_{E}(\cdot, 0) \geq \tilde{d}_{F}(\cdot, 0)$. We claim that

$$
\tilde{d}_{E} \geq \tilde{d}_{F} \text { on } \partial S \times\left(0, t^{*}\right) .
$$

Here and in the rest of the proof we may assume without loss of generality that $t^{*}$ is as small as needed (but still depending only on $\Delta$ ). To this aim, write $\partial S=\Gamma_{1} \cup \Gamma_{2}$, where $\Gamma_{1}:=\left\{d_{E}(\cdot, 0)=\eta_{1}\right\}$ and $\Gamma_{2}:=\left\{d_{E}(\cdot, 0)=\eta_{2}\right\}$. Since $d_{F}(\cdot, 0)+\Delta \leq$ $d_{E}(\cdot, 0)=\eta_{1}$ on $\Gamma_{1}$, we deduce

$$
\tilde{d}_{F} \leq d_{F}+\Delta \leq \eta_{1}+\chi_{N} \sqrt{t} \leq \eta_{1}^{\prime} \leq \tilde{d}_{E}
$$

on $\Gamma_{1} \times\left(0, t^{*}\right)$. Similarly one can show that the inequality $\tilde{d}_{E} \geq \tilde{d}_{F}$ holds on $\Gamma_{2} \times\left(0, t^{*}\right)$.

Again by (3.6) we have

$$
d_{E} \geq \frac{\eta_{1}^{\prime \prime}}{2}>0 \quad \text { in }\left\{d_{E}(\cdot, 0) \geq \eta_{1}^{\prime \prime}\right\} \times\left(0, t^{*}\right)
$$

and, observing that $d_{F}(\cdot, 0) \leq \eta_{2}^{\prime \prime}-\Delta$ in $\left\{d_{E}(\cdot, 0) \leq \eta_{2}^{\prime \prime}\right\}$,

$$
d_{F} \leq \frac{\eta_{2}^{\prime \prime}-\Delta}{2}<0 \quad \text { in }\left\{d_{E}(\cdot, 0) \leq \eta_{2}^{\prime \prime}\right\} \times\left(0, t^{*}\right) .
$$

In particular

$$
E(t) \subset \subset F(t) \quad \text { for } t \in\left(0, t^{*}\right) .
$$

We now claim that, setting

$$
S^{\prime \prime}:=\left\{x \in \mathbb{R}^{N}: \eta_{1}^{\prime \prime}<d_{E}(x, 0)<\eta_{2}^{\prime \prime}\right\},
$$

we have

$$
\tilde{d}_{E}=d_{E} \quad \text { and } \quad \tilde{d}_{F}=d_{F}+\Delta \quad \text { in } S^{\prime \prime} \times\left(0, t^{*}\right) .
$$

Indeed by (3.6) we have

$$
d_{E}(x, t) \geq \eta_{1}^{\prime \prime}-\chi_{N} \sqrt{t} \geq \eta_{1}+\chi_{N} \sqrt{t} \quad \text { for }(x, t) \in S^{\prime \prime} \times\left(0, t^{*}\right)
$$

and thus $\tilde{d}_{E}=d_{E}$ in $S^{\prime \prime} \times\left(0, t^{*}\right)$. The proof of the second identity in (3.10) is analogous.

Now we will use quite standard parabolic maximum principles, like in the proof of Lemma 3.2. Notice that

$$
\partial_{t} \tilde{d}_{E}=\sum_{t \in J}\left[\tilde{d}_{E}(\cdot, t+0)-\tilde{d}_{E}(\cdot, t-0)\right] d x+\partial_{t}^{d} \tilde{d}_{E},
$$


where $J$ is the (countable) set of times where $d_{E}$ possibly jumps and $\partial_{t}^{d} \tilde{d}_{E}$ is the diffuse part of the distributional derivative. Using for instance the chain rule proved in [4], in $S \times\left(0, t^{*}\right)$ we have that

$$
\partial_{t}^{d} \tilde{d}_{E}= \begin{cases}\frac{\chi_{N}}{2 \sqrt{t}} & \text { a.e. in }\left\{(x, t): \eta_{1}^{\prime}+\chi_{N} \sqrt{t}>d_{E}(x)\right\} \\ \partial_{t}^{d} d_{E} & \left|\partial_{t}^{d} d_{E}\right| \text {-a.e. in }\left\{(x, t): \eta_{1}^{\prime}+\chi_{N} \sqrt{t} \leq d_{E}(x)\right\} .\end{cases}
$$

An analogous formula holds for $\partial_{t}^{d} \tilde{d}_{F}$. Recalling that $\left(\operatorname{div} z_{E}\right)^{+}$and $\left(\operatorname{div} z_{F}\right)^{-}$belong to $L^{\infty}\left(S \times\left(0, t^{*}\right)\right)$ it follows that (possibly modifying $t^{*}$ )

$$
\partial_{t}^{d} \tilde{d}_{E} \geq \operatorname{div} z_{E} \quad \text { and } \quad \partial_{t}^{d} \tilde{d}_{F} \leq \operatorname{div} z_{F}
$$

in the sense of measures in $S \times\left(0, t^{*}\right)$. Note also that a.e. in $S \times\left(0, t^{*}\right)$

$$
z_{E} \in \partial \phi\left(\nabla \tilde{d}_{E}\right) \quad \text { and } \quad z_{F} \in \partial \phi\left(\nabla \tilde{d}_{F}\right) .
$$

Fix $p>N$ and set $\Psi(s):=\left(s^{+}\right)^{p}$ and $w:=\Psi\left(\tilde{d}_{F}-\tilde{d}_{E}\right)$. By (3.7) we have

$$
w=0 \quad \text { on } \partial S \times\left(0, t^{*}\right) .
$$

Using as before the chain rule for $B V$ functions, recalling (3.11) and the fact that the jump parts of $\partial_{t} \tilde{d}_{E}$ and $\partial_{t} \tilde{d}_{F}$ are nonnegative and nonpositive, respectively, we have

$$
\partial_{t} w \leq \Psi^{\prime}\left(\tilde{d}_{F}-\tilde{d}_{E}\right)\left(\partial_{t}^{d} \tilde{d}_{F}-\partial_{t}^{d} \tilde{d}_{E}\right) \leq \Psi^{\prime}\left(\tilde{d}_{F}-\tilde{d}_{E}\right)\left(\operatorname{div} z_{F}-\operatorname{div} z_{E}\right)
$$

in $S \times\left(0, t^{*}\right)$. Choose a cut-off function $\eta \in C_{c}^{\infty}\left(\mathbb{R}^{N}\right)$ such that $0 \leq \eta \leq 1$ and $\eta \equiv 1$ on $B_{1}$. For every $\varepsilon>0$ we set $\eta_{\varepsilon}(x):=\eta(\varepsilon x)$. Using (3.13) and (3.14), we have

$$
\begin{aligned}
\partial_{t} \int_{S} w \eta_{\varepsilon}^{p} d x \leq & \int_{S} \eta_{\varepsilon}^{p} \Psi^{\prime}\left(\tilde{d}_{F}-\tilde{d}_{E}\right)\left(\operatorname{div} z_{F}-\operatorname{div} z_{E}\right) \\
= & -\int_{S} \eta_{\varepsilon}^{p} \Psi^{\prime \prime}\left(\tilde{d}_{F}-\tilde{d}_{E}\right)\left(z_{F}-z_{E}\right) \cdot\left(\nabla \tilde{d}_{F}-\nabla \tilde{d}_{E}\right) d x+ \\
& p \int_{S} \eta_{\varepsilon}^{p-1} \Psi^{\prime}\left(\tilde{d}_{F}-\tilde{d}_{E}\right) \nabla \eta_{\varepsilon} \cdot\left(z_{F}-z_{E}\right) d x \\
\leq & p \int_{S} \eta_{\varepsilon}^{p-1} \Psi^{\prime}\left(\tilde{d}_{F}-\tilde{d}_{E}\right) \nabla \eta_{\varepsilon} \cdot\left(z_{F}-z_{E}\right) d x
\end{aligned}
$$

where we have also used the inequality $\left(z_{F}-z_{E}\right) \cdot\left(\nabla \tilde{d}_{F}-\nabla \tilde{d}_{E}\right) \geq 0$, which follows from (3.12) and the convexity of $\phi$. By Hölder Inequality and using the explicit expression of $\Psi$ and $\Psi^{\prime}$, we get

$$
\partial_{t} \int_{S} w \eta_{\varepsilon}^{p} d x \leq C p^{2}\left\|\nabla \eta_{\varepsilon}\right\|_{L^{p}\left(\mathbb{R}^{N}\right)}\left(\int_{S} w \eta_{\varepsilon}^{p} d x\right)^{1-\frac{1}{p}},
$$

for some constant $C>0$ depending only on the $L^{\infty}$-norms of $z_{E}$ and $z_{F}$. Since $w=0$ at $t=0$, a simple ODE argument then yields

$$
\int_{S} w \eta_{\varepsilon}^{p} d x \leq\left(C p\left\|\nabla \eta_{\varepsilon}\right\|_{L^{p}\left(\mathbb{R}^{N}\right)} t\right)^{p}
$$

for all $t \in\left(0, t^{*}\right)$. Observing that $\left\|\nabla \eta_{\varepsilon}\right\|_{L^{p}\left(\mathbb{R}^{N}\right)}^{p}=\varepsilon^{p-N}\|\nabla \eta\|_{L^{p}\left(\mathbb{R}^{N}\right)}^{p} \rightarrow 0$ and $\eta_{\varepsilon} \nearrow 1$ as $\varepsilon \rightarrow 0^{+}$, we conclude that $w=0$, and in turn $\tilde{d}_{E} \geq \tilde{d}_{F}$ in $S \times\left(0, t^{*}\right)$. In particular, by claim (3.10), we have shown that $d_{E} \geq d_{F}+\Delta$ in $S^{\prime \prime} \times\left(0, t^{*}\right)$. We finally claim that $\operatorname{dist}\left(E(t), F^{c}(t)\right) \geq \Delta$ for $t \in\left(0, t^{*}\right)$. To see this, fix $\varepsilon \geq 0$, and let let $x \in \partial E(t)$ and $y \in \partial F(t)$ be such that $\phi^{\circ}(x-y) \leq \operatorname{dist}\left(E(t), F^{c}(t)\right)+\varepsilon$. Note that by (3.8) and 
(3.9) we have $d_{E}(x, 0)<\eta_{1}^{\prime \prime}$ and $d_{E}(y, 0)>\eta_{2}^{\prime \prime}$. Thus there exists $z \in S^{\prime \prime} \cap[x, y]$, where $[x, y]$ denotes the segment joining $x$ and $y$. Since $d_{E}(\cdot, t) \geq d_{F}(\cdot, t)+\Delta$ in $S^{\prime \prime}$, we have

$$
\begin{aligned}
\operatorname{dist}\left(E(t), F^{c}(t)\right) \geq \phi^{\circ}(x-y)-\varepsilon=\phi^{\circ}( & x-z)+\phi^{\circ}(z-y)-\varepsilon \geq \\
& -d_{F}(z, t)+d_{E}(z, t)-\varepsilon \geq \Delta-\varepsilon .
\end{aligned}
$$

The claim follows by the arbitrariness of $\varepsilon$, and this concludes the proof of the theorem.

\section{Existence VIA Minimizing MOVEMents}

In this section we prove an existence result for the crystalline curvature flow, according to Definition 2.1. Such a solution is obtained via a variant of the AlmgrenTaylor-Wang minimizing movements scheme ([2]) introduced in [18, 16].

4.1. Minimizing movements. Let $E^{0} \subset \mathbb{R}^{N}$ be closed. Fix a time-step $h>0$ and set $E_{h}^{0}=E^{0}$. We then inductively define $E_{h}^{k+1}$ (for all $k \in \mathbb{N}$ ) according to the following procedure: If $E_{h}^{k} \neq \emptyset, \mathbb{R}^{N}$, then let $\left(u_{h}^{k+1}, z_{h}^{k+1}\right): \mathbb{R}^{N} \rightarrow \mathbb{R} \times \mathbb{R}^{N}$ satisfy

$$
\left\{\begin{array}{l}
-h \operatorname{div} z_{h}^{k+1}+u_{h}^{k+1}=d_{E_{h}^{k},} \\
z_{h}^{k+1} \in \partial \phi\left(\nabla u_{h}^{k+1}\right) \quad \text { a.e. in } \mathbb{R}^{N},
\end{array}\right.
$$

and set $E_{h}^{k+1}:=\left\{x: u_{h}^{k+1} \leq 0\right\}$. If either $E_{h}^{k}=\emptyset$ or $E_{h}^{k}=\mathbb{R}^{N}$, then set $E_{h}^{k+1}:=E_{h}^{k}$. We denote by $T_{h}^{*}$ the first discrete time $h k$ such that $E_{h}^{k}=\emptyset$, if such a time exists; otherwise we set $T_{h}^{*}=+\infty$.

In proposition 4.1 below we will show that this construction is well defined, since problem (4.1) admits a unique solution $u_{h}^{k+1}$ that is Lipschitz continuous. In particular, $E_{h}^{k+1}$ is a closed set for all $k$.

Before stating the main facts about the differential problem (4.1), we recall that given $z \in L^{\infty}\left(\mathbb{R}^{N} ; \mathbb{R}^{N}\right)$ with $\operatorname{div} z \in L_{\text {loc }}^{2}\left(\mathbb{R}^{N}\right)$ and $w \in B V_{\text {loc }}\left(\mathbb{R}^{N}\right) \cap L_{\text {loc }}^{2}\left(\mathbb{R}^{N}\right), z \cdot D w$ denotes the Radon measure associated with the linear functional

$$
L \varphi:=-\int_{\mathbb{R}^{N}} w \varphi \operatorname{div} z d x-\int_{\mathbb{R}^{N}} w z \cdot \nabla \varphi d x \quad \text { for all } \varphi \in C_{c}^{\infty}\left(\mathbb{R}^{N}\right),
$$

see [10].

Proposition 4.1. Let $g \in L_{\text {loc }}^{2}\left(\mathbb{R}^{N}\right)$. There exists a field $z \in L^{\infty}\left(\mathbb{R}^{N} ; W(0,1)\right)$ and a unique function $u \in B V_{l o c}\left(\mathbb{R}^{N}\right) \cap L_{\text {loc }}^{2}\left(\mathbb{R}^{N}\right)$ such that the pair $(u, z)$ satisfies

$$
\begin{cases}-h \operatorname{div} z+u=g & \text { in } \mathcal{D}^{\prime}\left(\mathbb{R}^{N}\right), \\ \phi^{\circ}(z) \leq 1 & \text { a.e. in } \mathbb{R}^{N}, \\ z \cdot D u=\phi(D u) & \text { in the sense of measures. }\end{cases}
$$

Moreover, for any $R>0$ and $v \in B V\left(B_{R}\right)$ with $\operatorname{Supp}(u-v) \Subset B_{R}$,

$$
\phi(D u)\left(B_{R}\right)+\frac{1}{2 h} \int_{B_{R}}(u-g)^{2} d x \leq \phi(D v)\left(B_{R}\right)+\frac{1}{2 h} \int_{B_{R}}(v-g)^{2} d x,
$$

and for every $s \in \mathbb{R}$ the set $E_{s}:=\left\{x \in \mathbb{R}^{N}: u(x) \leq s\right\}$ solves the minimization problem

$$
\min _{F \Delta E_{s} \Subset B_{R}} P_{\phi}\left(F ; B_{R}\right)+\frac{1}{h} \int_{F \cap B_{R}}(g(x)-s) d x .
$$


If $g_{1} \leq g_{2}$ and if $u_{1}, u_{2}$ are the corresponding solutions to (4.2) (with $g$ replaced by $g_{1}$ and $g_{2}$, respectively), then $u_{1} \leq u_{2}$.

Finally if in addition $g$ is Lipschitz with $\phi(\nabla g) \leq 1$, then the unique solution $u$ of (4.2) is also Lipschitz and satisfies $\phi(\nabla u) \leq 1$ a.e. in $\mathbb{R}^{N}$. As a consequence, (4.2) is equivalent to

$$
\left\{\begin{array}{l}
-h \operatorname{div} z+u=g, \\
z \in \partial \phi(\nabla u) \quad \text { a.e. in } \mathbb{R}^{N}
\end{array}\right.
$$

Proof. See [16, Theorem 2], [1, Theorem 3.3].

Remark 4.2 (Consistency with the ATW scheme). When $\partial E^{0}$ is bounded, the minimality property of the level sets stated above shows, in particular, that the sets $E_{h}^{k}$ are constructed according to the Almgren-Taylor-Wang scheme [2].

Since by the previous proposition $\phi\left(\nabla u_{h}^{k+1}\right) \leq 1$ a.e. in $\mathbb{R}^{N}$, one deduces, in particular, that

$$
\begin{array}{ll}
u_{h}^{k+1} \leq d_{E_{h}^{k+1}} & \text { in }\left\{x: \operatorname{dist}\left(x, E_{h}^{k+1}\right)>0\right\} \\
u_{h}^{k+1} \geq d_{E_{h}^{k+1}} & \text { in }\left\{x: \operatorname{dist}\left(x, E_{h}^{k+1}\right)<0\right\} .
\end{array}
$$

We are now in a position to define the time discrete evolutions. Precisely, we set

$$
\begin{aligned}
& E_{h}:=\left\{(x, t): x \in E_{h}^{[t / h]}\right\}, \\
& E_{h}(t):=E_{h}^{[t / h]}=\left\{x:(x, t) \in E_{h}\right\}, \\
& d_{h}(x, t):=d_{E_{h}(t)}(x), \\
& u_{h}(x, t):=u_{h}^{[t / h]}(x), \\
& z_{h}(x, t):=z_{h}^{[t / h]}(x),
\end{aligned}
$$

where $[\cdot]$ stands for the integer part of its argument.

Remark 4.3 (Discrete comparison principle). The last part of Proposition 4.1 clearly implies that the scheme is monotone, that is, the discrete evolutions satisfy the comparison principle. More precisely, if $E^{0} \subseteq F^{0}$ are closed sets and if we denote by $E_{h}$ and $F_{h}$ the discrete evolutions with initial datum $E^{0}$ and $F^{0}$, respectively, then $E_{h} \subseteq F_{h}$.

4.2. Comparison with the Wulff shape. In this subsection, we exploit Remark 4.3 to compare the discrete evolutions (4.5) with the minimizing movements of the Wulff shape and derive an estimate, which will be useful in the convergence analysis. The evolution starting from a Wulff shape $W(0, R)$ is explicitly known. Indeed, from [16, Appendix B, Eq. (39)], the solution of (4.2), with $g$ replaced by $d_{W(0, R)}=\phi^{\circ}-R$, is given by $\phi_{h}^{\circ}-R$, where

$$
\phi_{h}^{\circ}(x):= \begin{cases}\sqrt{h} \frac{2 N}{\sqrt{N+1}} & \text { if } \phi^{\circ}(x) \leq \sqrt{h(N+1)}, \\ \phi^{\circ}(x)+h \frac{N-1}{\phi^{\circ}(x)} & \text { else. }\end{cases}
$$

It follows that if $E^{0}=W(0, R)$, one has $E_{h}(t)=W\left(0, r_{h}^{R}(t)\right)$ for a function $r_{h}^{R}$ that satisfies

$$
r_{h}^{R}(h)=\frac{R+\sqrt{R^{2}-4 h(N-1)}}{2}
$$


if $h \leq R^{2} /(4(N+1))$. In particular,

$$
r_{h}^{R}(h) \geq \sqrt{R^{2}-4 h(N-1)}
$$

for the same $h$ 's. By iteration, we have $r_{h}^{R}(t) \geq \sqrt{R^{2}-4 t(N-1)} \geq \frac{R}{\sqrt{2}}$ for $0 \leq t \leq$ $R^{2} /(8(N-1))$ and $h \leq R^{2} /(8(N+1))$. Since $r_{h}^{R}(t)=R$ for $t \in[0, h)$, we infer

$$
r_{h}^{R}(t) \geq \sqrt{R^{2}-4 t(N-1)}
$$

for $0 \leq t \leq R^{2} /(8(N+1))$ and for all $h$.

Now we return to the motion from an arbitrary set $E^{0}$. If for some $(x, t) \in$ $\mathbb{R}^{N} \times\left[0, T_{h}^{*}\right)$ we have $d_{h}(x, t)>R$, then $W(x, R) \cap E_{h}(t)=\emptyset$. Hence, by the comparison principle stated in Remark 4.3 and by (4.7) we have

$$
d_{h}(x, s) \geq \sqrt{R^{2}-4(N-1)(s-t+h)}
$$

for $t<s$ and $s+h-t<R^{2} /(8(N+1))$.

By letting $R \nearrow d_{h}(x, t)$ we obtain

$$
d_{h}(x, s) \geq \sqrt{d_{h}^{2}(x, t)-4(N-1)(s-t+h)}
$$

for $t<s$ and $s+h-t<d_{h}^{2}(x, t) /(8(N+1))$.

4.3. Convergence of the scheme. Up to a subsequence we have

$$
E_{h_{l}} \stackrel{\mathcal{K}}{\longrightarrow} E \quad \text { and } \quad\left({\stackrel{\circ}{h_{l}}}_{l}\right)^{c} \stackrel{\mathcal{K}}{\longrightarrow} A^{c}
$$

for a suitable closed sets $E$ and a suitable open set $A \subset E$. Define $E(t)$ and $A(t)$ as in (4.5).

Observe that if $E(t)=\emptyset$ for some $t \geq 0$, then (4.8) implies that $E(s)=\emptyset$ for all $s \geq t$ so that we can define, as in Definition 2.1 the extinction time $T^{*}$ of $E$, and similarly the extinction time $T^{\prime *}$ of $A^{c}$. Notice that at least one between $T^{*}$ and $T^{\prime *}$ is $+\infty$. Possibly extracting a further subsequence, we have the following result:

Proposition 4.4. There exists a countable set $\mathcal{N} \subset(0,+\infty)$ such that $d_{h_{l}}(\cdot, t)^{+} \rightarrow$ $\operatorname{dist}(\cdot, E(t))$ and $d_{h_{l}}(\cdot, t)^{-} \rightarrow \operatorname{dist}\left(\cdot, A^{c}\right)$ locally uniformly for all $t \in(0,+\infty) \backslash \mathcal{N}$.

Moreover, for every $x \in \mathbb{R}^{N}$ the functions $\operatorname{dist}(x, E(\cdot))$ and $\operatorname{dist}\left(x, A^{c}\right)$ are left continuous and are right lower semicontinuous. Equivalently, the functions $E(\cdot)$ and $A^{c}$ are left continuous and are right upper semicontinuous with respect to the Kuratowski convergence. Finally, $E(0)=E^{0}$ and $A(0)=E^{0}$.

Proof. By the Ascoli-Arzelà Theorem and a standard diagonal argument, we may extract a further (not relabeled) subsequence such that $d_{h_{l}}(\cdot, t) \rightarrow d(\cdot, t)$ locally uniformly for all $t \in \mathbb{Q} \cap(0,+\infty)$, where $d(\cdot, t)$ is either a Lipschitz function or infinite everywhere. In the latter case, either $d(\cdot, t) \equiv+\infty$ or $d(\cdot, t) \equiv-\infty$.

We observe that for all $t \in\left(0, T^{*}\right) \cap \mathbb{Q}$ we have $d(\cdot, t)<+\infty$. To see this we argue by contradiction assuming that for every $x \in \mathbb{R}^{N}$ and for every $M>0$ we have $d_{h_{l}}(x, t)>M$ for all $l$ large enough. We may now apply (4.8) to deduce that there exists a right interval $\left(t, t^{\prime}\right)$ independent of $l$ such that $d_{h_{l}}(x, s)>\frac{M}{2}$ for $l$ large enough and for all $s \in\left(t, t^{\prime}\right)$; that is, $d_{h_{l}}(\cdot, s) \rightarrow+\infty$ for all $s \in\left(t, t^{\prime}\right)$. This in turn would imply $E(s)=\emptyset$ for all $s \in\left(t, t^{\prime}\right)$, which is impossible since $t<T^{*}$. A similar argument shows that for all $t \in\left(0, T^{\prime *}\right)$ we have $d(\cdot, t)>-\infty$. 
Let $x \in \mathbb{R}^{N}$ and $t>0$ be such that $\limsup _{l} d_{h_{l}}(x, t)=: R>0$. Then, given $0<R^{\prime}<R$, we have $d_{h_{l}}(x, t) \geq R^{\prime}$ for infinitely many $l$. By (4.8), for

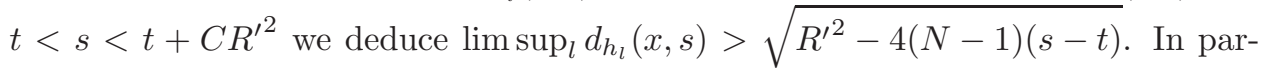
ticular if $s \in \mathbb{Q} \cap\left(t, t+C R^{2}\right)$, then it follows $d(x, s) \geq \sqrt{R^{2}-4(N-1)(s-t)}$. If, in addition, also $t \in \mathbb{Q}$, then we have $d(x, s) \geq \sqrt{d(x, t)^{2}-4(N-1)(s-t)}$. Now let $x \in \mathbb{R}^{N}$ and $t \geq 0$, and assume $R:=\limsup _{s \in \mathbb{Q}, s \searrow t} d(x, t)>0$. Consider a sequence of rational numbers $s_{k} \searrow t$ such that $\lim _{k} d\left(x, s_{k}\right)=R$. For $s>t$ rational and close enough to $t$, if $k$ is sufficiently large, then $s_{k}<s$ and $d(x, s) \geq \sqrt{d\left(x, s_{k}\right)^{2}-4(N-1)\left(s-s_{k}\right)}$. Sending $k$ to infinity it follows $d(x, s) \geq$ $\sqrt{R^{2}-4(N-1)(s-t)}$ so that $\liminf _{s \in \mathbb{Q}, s \searrow t} d(x, t) \geq R$. Hence $d(x, \cdot)$ has a right limit at $t$. The same conclusion holds if $\liminf _{s \in \mathbb{Q}, s \searrow t} d(x, t)<0$, with the same proof. We deduce that the $d$ admits a right limit (locally uniformly in space) at any $t \geq 0$. A similar argument shows that $d$ also admits a left limit at any $t>0$. Moreover, arguing similarly and using (4.8) again, we can show that

$$
\begin{aligned}
d(x, t+0)^{ \pm} & \geq \limsup _{l \rightarrow \infty, s \rightarrow t} d_{h_{l}}(x, s)^{ \pm} \\
& \geq \liminf _{l \rightarrow \infty, s \rightarrow t} d_{h_{l}}(x, s)^{ \pm} \geq d(x, t-0)^{ \pm} .
\end{aligned}
$$

Let $\mathcal{N}$ be the set of all times $t$ such that the left and right limits of $d$ differ at $(x, t)$, for some $x \in \mathbb{R}^{N}$ (we also assume $0 \in \mathcal{N}$ ). Notice that $\mathcal{N}$ is countable, since it can be written as the union over $k \in \mathbb{N}$ and $x \in \mathbb{Q}^{N}$ of the times such that the gap between the right and left limit of $d(x, \cdot)$ is larger than $1 / k$ (which for fixed $k$ and $x$ cannot have cluster points). We denote by $d(x, t)$ the common value of the right and left limits of $d(x, \cdot)$ at $t \notin \mathcal{N}$.

By (4.9) we immediately have that $\lim _{l \rightarrow \infty} d_{h_{l}}(\cdot, t)=d(\cdot, t)$ for all $t \notin \mathcal{N}$. We now show that for $t \notin \mathcal{N}$, we have $d(\cdot, t)^{+}=\operatorname{dist}(\cdot, E(t))$. This is equivalent to showing that $E(t)$ coincides with the Kuratowski limit $K$ of $E_{h_{l}}(t)$, since $d(\cdot, t)^{+}=\operatorname{dist}(\cdot, K)$. Clearly, $K \subseteq E(t)$. Conversely, if $x \notin K$, then $d(x, t)^{+}=: R>0$. Since $d$ is continuous at $t$, we may find $\varepsilon$ so small that $\lim _{l \rightarrow \infty} d_{h_{l}}(x, t-\varepsilon) \geq d(x, t-\varepsilon)>R / 2$ and in turn, by (4.8), $W(x, R / 4) \times[t-\varepsilon, t+\varepsilon] \cap E_{h_{l}}=\emptyset$ for $l$ large enough. Thus $x \notin E(t)$, showing that $E(t)=K$ and $d(x, t)^{+}=\operatorname{dist}(x, E(t))$. A similar argument yields that $d(x, t)^{-}=\operatorname{dist}\left(x, A^{c}\right)$.

Always by (4.8), one can easily prove that $E(0) \subseteq E^{0}$. Since $E_{h_{l}}(0)=E^{0}$ for all $l$, we infer the equality $E(0)=E^{0}$. Symmetrically, one can show that $A(0)=E^{0}$.

Finally, we prove the continuity properties of $E(t)$. The right upper semicontinuity with respect to the Kuratowski convergence is a consequence of the fact that $E$ is closed. Let us prove now the left continuity. To this aim, denote by $\hat{K}$ the Kuratowski limit of $E(s)$ as $s \nearrow t$. Clearly $\hat{K} \subseteq E(t)$. Let now $x \notin \hat{K}$. Then $\lim _{s \nearrow_{t}} \operatorname{dist}(x, E(s))=\operatorname{dist}(x, \hat{K})=: R>0$. Arguing exactly as before we may choose $\varepsilon$ so small that $\liminf _{l} \operatorname{dist}\left(x, E_{h_{l}}(t-\varepsilon)\right) \geq \operatorname{dist}(x, E(t-\varepsilon))>R / 2$ and $W(x, R / 4) \times[t-\varepsilon, t+\varepsilon] \cap E_{h_{l}}=\emptyset$ for all $l$ large enough, so that $x \notin E(t)$. Hence $\hat{K}=E(t)$. This establishes the Kuratowski left-continuity of $E(\cdot)$ and concludes the proof of the proposition.

Theorem 4.5. E is a supersolution in the sense of Definition 2.1 with initial datum $E^{0}$, while $A$ is a subsolution with initial datum $E^{0}$. 
Proof. Points (a), (b) and (c) of Definition 2.1 follow from Proposition 4.4. It remains to show $(d)$. Possibly extracting a further subsequence and setting $z_{h_{l}}(\cdot, t):=$ 0 for $t>T_{h_{l}}^{*}$ if $T_{h_{l}}^{*}<T^{*}$, we may assume that $z_{h_{l}}$ converges weakly-* in $L^{\infty}\left(\mathbb{R}^{N} \times\right.$ $\left.\left(0, T^{*}\right)\right)$ to some vector-field $z$ satisfying $\phi^{\circ}(z) \leq 1$ almost everywhere. Recall that by (4.4) we have $u_{h}^{k+1} \leq d_{E_{h}^{k+1}}$, whenever $d_{E_{h}^{k+1}} \geq 0$. In turn, it follows from (4.1) that

$$
\operatorname{div} z_{h}^{k+1} \leq \frac{d_{E_{h}^{k+1}}-d_{E_{h}^{k}}}{h} \quad \text { a.e. on }\left\{d_{E_{h}^{k+1}} \geq 0\right\}
$$

Consider a nonnegative test function $\eta \in C_{c}^{\infty}\left(\left(\mathbb{R}^{N} \times\left(0, T^{*}\right)\right) \backslash E\right)$. If $l$ is large enough, then the distance of the support of $\eta$ from $E_{h_{l}}$ is bounded away from zero. In particular, $d_{h_{l}}$ is finite and positive on Supp $\eta$. We deduce from (4.10) that

$$
\begin{aligned}
& \iint \eta(x, t)\left(\frac{d_{h_{l}}\left(x, t+h_{l}\right)-d_{h_{l}}(x, t)}{h_{l}}-\operatorname{div} z_{h_{l}}\left(x, t+h_{l}\right)\right) d t d x \\
& =-\iint\left(\frac{\eta(x, t)-\eta\left(x, t-h_{l}\right)}{h_{l}} d_{h_{l}}(x, t)-z_{h_{l}}\left(x, t+h_{l}\right) \cdot \nabla \eta(x, t)\right) d t d x \geq 0 .
\end{aligned}
$$

Passing to the limit $l \rightarrow \infty$ we obtain (2.4).

Next, we establish an upper bound for $\operatorname{div} z_{h_{l}}$ away from $E_{h_{l}}$. To this aim observe that

$$
d_{E_{h}^{k}}=\min _{y \in E_{h}^{k}} \phi^{\circ}(\cdot-y)
$$

so that, by (4.1) and the comparison principle stated at the end of Proposition 4.1 .

$$
u_{h}^{k+1} \leq \min _{y \in E_{h}^{k}} \phi_{h}^{\circ}(\cdot-y)
$$

where $\phi_{h}^{\circ}$ is given in (4.6). Thus, if $d_{E_{h}^{k}}(x) \geq R>0$, then

$$
u_{h}^{k+1}(x) \leq \min _{y \in E_{h}^{k}} \phi^{\circ}(x-y)+h \frac{N-1}{R}=d_{E_{h}^{k}}(x)+h \frac{N-1}{R},
$$

provided $h \leq R^{2} /(N+1)$. As a consequence of (4.1), we obtain

$$
\operatorname{div} z_{h}^{k+1} \leq \frac{N-1}{R} \quad \text { a.e. in }\left\{x: d_{E_{h}^{k}}(x) \geq R\right\} .
$$

It is then easy to deduce from the convergence properties of $E_{h_{l}}$ and $d_{h_{l}}$ that

$$
\operatorname{div} z \leq \frac{N-1}{R} \quad \text { in }\left\{(x, t) \in \mathbb{R}^{N} \times\left(0, T^{*}\right): d(x, t)>R\right\}
$$

in the sense of distributions. It follows that $\operatorname{div} z$ is a Radon measure in $\mathbb{R}^{N} \times$ $\left(0, T^{*}\right) \backslash E$, and $(\operatorname{div} z)^{+} \in L^{\infty}\left(\left\{(x, t) \in \mathbb{R}^{N} \times\left(0, T^{*}\right): d(x, t) \geq \delta\right\}\right)$ for every $\delta>0$.

We now provide a lower (h-dependent) bound for $\operatorname{div} z_{h_{l}}$. To this aim, note that if $d_{E_{h}^{k}}(x)=: R>0$, then $d_{E_{h}^{k}} \geq R-\phi^{\circ}(\cdot-x)$. Thus, by comparison as before,

$$
u_{h}^{k+1}(x) \geq R-\phi_{h}^{\circ}(0)=R-\sqrt{h} \frac{2 N}{\sqrt{N+1}} .
$$

In turn, by (4.1), we deduce

$$
\operatorname{div} z_{h}^{k+1} \geq-\frac{1}{\sqrt{h}} \frac{2 N}{\sqrt{N+1}} \quad \text { a.e. in }\left\{x: d_{E_{h}^{k}}(x)>0\right\} .
$$


Combining the above inequality with (4.11) and using (4.1) again, we deduce that for all $t \in\left(0, T^{*}\right) \backslash \mathcal{N}$ (where recall that $\mathcal{N}$ is introduced in Proposition 4.4) and any $\delta>0$

$$
\left\|u_{h_{l}}(\cdot, t)-d_{h_{l}}\left(\cdot, t-h_{l}\right)\right\|_{L^{\infty}\left(\left\{x: d_{h_{l}}\left(x, t-h_{l}\right) \geq \delta\right\}\right)} \leq \sqrt{h} \frac{2 N}{\sqrt{N+1}},
$$

provided that $l$ is large enough. In particular, recalling the convergence properties of $E_{h_{l}}$ and $d_{h_{l}}$ (see also (4.9l)), we deduce that

$$
u_{h_{l}} \rightarrow d \quad \text { a.e. in } \mathbb{R}^{N} \times\left(0, T^{*}\right) \backslash E,
$$

with the sequence $\left\{u_{h_{l}}\right\}$ locally (in space and time) uniformly bounded.

Consider now, as before, a nonnegative test function $\eta \in C_{c}^{\infty}\left(\left(\mathbb{R}^{N} \times\left(0, T^{*}\right)\right) \backslash E\right)$. Then, recalling (4.12), we have by lower semicontinuity

$$
\iint \phi(\nabla d) \eta d x d t \leq \liminf _{l} \iint \phi\left(\nabla u_{h_{l}}\right) \eta d x d t=\liminf _{l} \iint\left(z_{h_{l}} \cdot \nabla u_{h_{l}}\right) \eta d x d t .
$$

On the other hand,

$$
\iint\left(z_{h_{l}} \cdot \nabla u_{h_{l}}\right) \eta d x d t=\iint\left(z_{h_{l}} \cdot \nabla d\right) \eta d x d t+\iint z_{h_{l}} \cdot \nabla\left(u_{h_{l}}-d\right) \eta d x d t,
$$

with

Hence, we obtain

$$
\iint\left(z_{h_{l}} \cdot \nabla d\right) \eta d x d t \stackrel{l \rightarrow \infty}{\longrightarrow} \iint(z \cdot \nabla d) \eta d x d t .
$$

$$
\iint \phi(\nabla d) \eta d x d t \leq \iint(z \cdot \nabla d) \eta d x d t
$$

provided we show that

$$
\lim _{l} \iint z_{h_{l}} \cdot \nabla\left(u_{h_{l}}-d\right) \eta d x d t=0
$$

For each $t$, set

$$
m_{l}(t):=\min _{x \in \operatorname{Supp} \eta(\cdot, t)}\left(u_{h_{l}}(x, t)-d(x, t)\right), \quad M_{l}(t):=\max _{x \in \operatorname{Supp} \eta(\cdot, t)}\left(u_{h_{l}}(x, t)-d(x, t)\right) .
$$

Recall that these quantities are uniformly bounded and converge to 0 at all $t \notin \mathcal{N}$.

Then, we can write

$$
\begin{aligned}
\iint z_{h_{l}} \cdot \nabla\left(u_{h_{l}}-d\right) \eta & d x d t=\iint z_{h_{l}} \cdot \nabla\left(u_{h_{l}}-d-m_{l}\right) \eta d x d t \\
= & -\iint\left(u_{h_{l}}-d-m_{l}\right)\left(z_{h_{l}} \cdot \nabla \eta+\eta \operatorname{div} z_{h_{l}}\right) d x d t
\end{aligned}
$$

For $l$ large enough, since the support of $\eta$ is at positive distance from $E$ there exists $\delta>0$ such that $d_{h_{l}} \geq \delta$ everywhere on this support, so that $\operatorname{div} z_{h_{l}} \leq(N-1) / \delta$. It follows that

$$
-\iint\left(u_{h_{l}}-d-m_{l}\right) \eta \operatorname{div} z_{h_{l}} d x d t \geq-\frac{N-1}{\delta} \iint\left(u_{h_{l}}-d-m_{l}\right) \eta d x d t \stackrel{l \rightarrow \infty}{\longrightarrow} 0,
$$

thanks also to (4.12). Recalling (4.15), we can conclude that

$$
\liminf _{l} \iint z_{h_{l}} \cdot \nabla\left(u_{h_{l}}-d\right) \eta d x d t \geq 0 .
$$

In the same way, writing now

$$
\iint z_{h_{l}} \cdot \nabla\left(u_{h_{l}}-d\right) \eta d x d t=\iint z_{h_{l}} \cdot \nabla\left(u_{h_{l}}-d-M_{l}\right) \eta d x d t
$$


and using $u_{h_{l}}-d-M_{l} \leq 0$ a.e. on Supp $\eta$, one can show that

$$
\limsup _{l} \iint z_{h_{l}} \cdot \nabla\left(u_{h_{l}}-d\right) \eta d x d t \leq 0
$$

so that (4.14) follows. In turn, (4.13) holds, that is, $\phi(\nabla d) \leq z \cdot \nabla d$ a.e. in $\mathbb{R}^{N} \times\left(0, T^{*}\right) \backslash E$. On the other hand, recalling that $\phi^{\circ}(z) \leq 1$ a.e. in $\mathbb{R}^{N} \times\left(0, T^{*}\right)$, we have

$$
z \cdot \nabla d \leq \phi(\nabla d)
$$

a.e. in $\mathbb{R}^{N} \times\left(0, T^{*}\right)$. We conclude that $\phi(\nabla d)=z \cdot \nabla d$ and, in turn, $z \in \partial \phi(\nabla d)$ a.e. in $\mathbb{R}^{N} \times\left(0, T^{*}\right) \backslash E$. This concludes the proof that $E$ is a supersolution. The proof that $A$ is a subsolution is identical.

Corollary 4.6. Let $u^{0}$ be a bounded, uniformly continuous in $\mathbb{R}^{N}$. Then for all $s \in \mathbb{R}$ but a countable number, the minimizing movement scheme starting from $E_{s}^{0}=\left\{u^{0} \leq s\right\}$ converges to the unique solution of the curvature flow in the sense of Definition 2.1, with initial datum $E_{s}^{0}$.

Proof. The arguments are standard and rely on the comparison theorem 3.3 The bad (countable set) is the set of levels for which "fattening" occurs, that is, $|E \backslash A|>$ 0 . Observe that from Theorem 3.3. one easily shows the existence of a unique levelset solution $u(x, t)$ starting from $u^{0}$, which shares the same spatial modulus of continuity and is also uniformly continuous in time (see for instance [19, Subsection 6.3]).

\section{Conclusion ANd Perspectives}

In this note we have shown the existence and uniqueness of a mean curvature flow (namely, the "natural" flow by mean curvature along the Cahn-Hoffmann vector field) with a technique which does not require any type of regularity on the surface tension, and thus have provided the first sound definition of a crystalline curvature flow in any dimension. It does not require that the initial surface is bounded and applies, in particular, also to the case of graphs. The uniqueness result is based on a very standard parabolic comparison principle. The general approach, based on the fact that the level sets of the distance functions have nonincreasing curvatures as the distance increases (as was exploited as early as in [39 in the viscosity setting), can quite probably be used in more general situations, and even maybe for motions which are not necessarily variational. However, it should need substantial adaption. For instance, if replacing the mobility $m=\phi^{\circ}$ in our approach by other (convex) functions is in principle easy (it is enough to consider, for the distance functions, the $m$-distance function instead of the $\phi^{\circ}$-distance), in the nonsmooth case it yields difficulties which still require further investigation. Indeed, if $m$ is smooth and $\phi$ is not, then it will not be true anymore that the level sets of the distance function have globally bounded curvature as the distance increases, so that Definition 2.1 needs to be changed. It is not yet clear what assumption on $(\operatorname{div} z)^{ \pm}$is then useful

in order to be able to derive both existence and uniqueness. This is a subject for future study. 


\section{REFERENCES}

[1] L. Almeida, A. Chambolle, and M. Novaga. Mean curvature flow with obstacles. Ann. Inst. H. Poincaré Anal. Non Linéaire, 29(5):667-681, 2012.

[2] F. Almgren, J. E. Taylor, and L.-H. Wang. Curvature-driven flows: a variational approach. SIAM J. Control Optim., 31(2):387-438, 1993.

[3] Fred Almgren and Jean E. Taylor. Flat flow is motion by crystalline curvature for curves with crystalline energies. J. Differential Geom., 42(1):1-22, 1995.

[4] L. Ambrosio and G. Dal Maso. A general chain rule for distributional derivatives. Proc. Amer. Math. Soc., 108(3):691-702, 1990.

[5] L. Ambrosio, N. Fusco, and D. Pallara. Functions of bounded variation and free discontinuity problems. Oxford Mathematical Monographs. The Clarendon Press Oxford University Press, New York, 2000.

[6] Luigi Ambrosio and Halil Mete Soner. Level set approach to mean curvature flow in arbitrary codimension. J. Differential Geom., 43(4):693-737, 1996.

[7] S. Angenent, T. Ilmanen, and D. L. Chopp. A computed example of nonuniqueness of mean curvature flow in $\mathbf{R}^{3}$. Comm. Partial Differential Equations, 20(11-12):1937-1958, 1995.

[8] Sigurd Angenent and Morton E. Gurtin. Multiphase thermomechanics with interfacial structure. II. Evolution of an isothermal interface. Arch. Rational Mech. Anal., 108(4):323-391, 1989.

[9] Sigurd Angenent and Morton E. Gurtin. Multiphase thermomechanics with interfacial structure. II. Evolution of an isothermal interface. Arch. Rational Mech. Anal., 108(4):323-391, 1989.

[10] G. Anzellotti. Pairings between measures and bounded functions and compensated compactness. Ann. Mat. Pura Appl. (4), 135:293-318 (1984), 1983.

[11] G. Barles, H. M. Soner, and P. E. Souganidis. Front propagation and phase field theory. SIAM J. Control Optim., 31(2):439-469, 1993.

[12] G. Bellettini, V. Caselles, and M. Novaga. The total variation flow in $\mathbb{R}^{N}$. J. Differential Equations, 184(2):475-525, 2002.

[13] G. Bellettini, M. Novaga, and M. Paolini. On a crystalline variational problem. I. First variation and global $L^{\infty}$ regularity. Arch. Ration. Mech. Anal., 157(3):165-191, 2001.

[14] Giovanni Bellettini, Vicent Caselles, Antonin Chambolle, and Matteo Novaga. Crystalline mean curvature flow of convex sets. Arch. Ration. Mech. Anal., 179(1):109-152, 2006.

[15] Kenneth A. Brakke. The motion of a surface by its mean curvature, volume 20 of Mathematical Notes. Princeton University Press, Princeton, N.J., 1978.

[16] Vicent Caselles and Antonin Chambolle. Anisotropic curvature-driven flow of convex sets. Nonlinear Anal., 65(8):1547-1577, 2006.

[17] A. Chambolle and M. Novaga. Existence and uniqueness for planar anisotropic and crystalline curvature flow. In L. Ambrosio, Y. Giga, P. Rybka, and Y. Tonegawa, editors, Variational Methods for Evolving Objects, volume 67 of Advanced Studies in Pure Mathematics, pages 87-113. Mathematical Society of Japan, 2015.

[18] Antonin Chambolle. An algorithm for mean curvature motion. Interfaces Free Bound., 6(2):195-218, 2004.

[19] Antonin Chambolle, Massimiliano Morini, and Marcello Ponsiglione. Nonlocal curvature flows. Arch. Ration. Mech. Anal., 2015.

[20] Antonin Chambolle and Matteo Novaga. Approximation of the anisotropic mean curvature flow. Math. Models Methods Appl. Sci., 17(6):833-844, 2007.

[21] Yun Gang Chen, Yoshikazu Giga, and Shun'ichi Goto. Uniqueness and existence of viscosity solutions of generalized mean curvature flow equations. J. Differential Geom., 33(3):749-786, 1991.

[22] Tokuhiro Eto, Yoshikazu Giga, and Katsuyuki Ishii. An area-minimizing scheme for anisotropic mean-curvature flow. Adv. Differential Equations, 17(11-12):1031-1084, 2012.

[23] Lawrence C. Evans. Convergence of an algorithm for mean curvature motion. Indiana Univ. Math. J., 42(2):533-557, 1993. 
[24] Lawrence C. Evans and Joel Spruck. Motion of level sets by mean curvature. I. J. Differential Geom., 33(3):635-681, 1991.

[25] Lawrence C. Evans and Joel Spruck. Motion of level sets by mean curvature. II. Trans. Amer. Math. Soc., 330(1):321-332, 1992.

[26] Irene Fonseca and Stefan Müller. A uniqueness proof for the Wulff theorem. Proc. Roy. Soc. Edinburgh Sect. A, 119(1-2):125-136, 1991.

[27] Mi-Ho Giga and Yoshikazu Giga. Evolving graphs by singular weighted curvature. Arch. Rational Mech. Anal., 141(2):117-198, 1998.

[28] Mi-Ho Giga and Yoshikazu Giga. Generalized motion by nonlocal curvature in the plane. Arch. Ration. Mech. Anal., 159(4):295-333, 2001.

[29] Mi-Ho Giga, Yoshikazu Giga, and Norbert Požár. Periodic total variation flow of nondivergence type in $\mathbb{R}^{n}$. J. Math. Pures Appl. (9), 102(1):203-233, 2014.

[30] Yoshikazu Giga and Morton E. Gurtin. A comparison theorem for crystalline evolution in the plane. Quart. Appl. Math., 54(4):727-737, 1996.

[31] Yoshikazu Giga, Morton E. Gurtin, and José Matias. On the dynamics of crystalline motions. Japan J. Indust. Appl. Math., 15(1):7-50, 1998.

[32] Matthew A. Grayson. A short note on the evolution of a surface by its mean curvature. Duke Math. J., 58(3):555-558, 1989.

[33] Morton E. Gurtin. Thermomechanics of evolving phase boundaries in the plane. Oxford Mathematical Monographs. The Clarendon Press, Oxford University Press, New York, 1993.

[34] Tom Ilmanen. Elliptic regularization and partial regularity for motion by mean curvature. Mem. Amer. Math. Soc., 108(520):x+90, 1994.

[35] Tim B. Laux and Felix Otto. Convergence of the thresholding scheme for multi-phase meancurvature flow. Preprint, Max Planck Institute for Mathematics in the Sciences, 2015.

[36] S. Luckhaus and T. Sturzenhecker. Implicit time discretization for the mean curvature flow equation. Calc. Var. Partial Differential Equations, 3(2):253-271, 1995.

[37] J. S. Moll. The anisotropic total variation flow. Math. Ann., 332(1):177-218, 2005.

[38] Stanley Osher and James A. Sethian. Fronts propagating with curvature-dependent speed: algorithms based on Hamilton-Jacobi formulations. J. Comput. Phys., 79(1):12-49, 1988.

[39] Halil Mete Soner. Motion of a set by the curvature of its boundary. J. Differential Equations, 101(2):313-372, 1993.

[40] Jean E. Taylor. Crystalline variational problems. Bull. Amer. Math. Soc., 84(4):568-588, 1978. 